\title{
Bibliographie über Grundtvig-Literatur in nichtskandinavischen Sprachen
}

\author{
Von Eberhard Harbsmeier
}

(1970)

\section{Von Grundtvig selbst in fremden Sprachen verfasste Texte}

Bibliotheca Anglo-Saxonica. Prospectus and Proposals of a subscription, for the publication of most valuable anglo-saxon manuscripts, illustrative of the early poetry and literature of our language. Most of which have never been printed. London 1830. 15S. 2. udg. 1831.

Rückblick auf sein Leben. Grundtvig Studier 58, 74-83.

(Rezension von) History of the Northmen, or Danes and Normans, from the Earliest Times to the Conquest of England by William of Normandy. By Henry Wheaton. 1831. The Westminster Review. Vol XV, 442-457.

An Stolberg. ("Geboren bin ich unter Seelands Eichen ..."); Poetiske Skrifter III, 77-83. cf. Lehmann, Grundtvig. S. 30 f. Deutsche Ausgabe.

Martin Luther. („Seyd gegrüsst, Ihr Martins Söhne ...“); Poetiske Skrifter IV, 475.

To Professor Whewell at Cambridge. ("Lines in books, or lines in hand ..."), Poetiske Skrifter V, 500-501.

Melrose Abbey and Abbotsford. (The dirge of Scotland in a word ...), Poetiske Skrifter VI, 470.

\section{Übersetzungen a) ins Englische}

William and Mary Howitt: The Literature and Romance of Northern Europe. 2 vls. London 1852. II, 164ff. (Song of Praise - The Mother-Tongue).

The Hymnes of Denmark. Tr. by Gilbert Tait (pseud.). London 1868. 153-155, 183-186, 207., 213f. Faith, Hope and Charity - Autumn - Sabbath Morning - Baptism; 153-155: Til klart Guds Aasyn vi skal se; 183-186: Nu falmer Skoven; 107f. Søndag Morgen fra de Døde; 213. Kommer hid kun med de Smaa.

In Denmark I was born ... A little book of Danish Verse, selected and translated by R.P. Keigwin. Copenhagen 1948. 37f, 39, 41. (The Church-Bell (37f) - Inscription on The Monument in Odde Churchyard (39) - Lullaby (Sov sødt, Barnlille!) (41).

John Volk: Songs and Poems in Danish and English. New York 1903. (The 
Church-bell - Jesus Streches forth his Hand (Herren strækker ud sin Arm).

The Easter-Lily. By Bishop N. F. S. Grundtvig. Translated by Alexander Marlowe Blair. Nebraska 1919.

Christian Science Hymnal, Boston 1932. Long Hast Thou Stood, O Church of God (Kirken, den er et gammelt Hus) Love Is Life's True Crown and Glory (Kærlighed er Lysets Kilde) Suffer the Children to Come to $\mathrm{Me}$ (Lille Guds Barn) The Lord Is in His Holy Place.

The American Scandinavian Review III, 1942, s. 228 (Denmarks Consolation, Tr. by S. F. Damon).

A Second Book of Danish Verse. Tr. by Charles Wharton Stork. With af foreword by Johannes V. Jensen. Princeton 1947. S. 6-9 (Niels Ebbesen). Selections from "Shall the Lutheran Reformation really be continued?" Translated by Valdemar S. Jensen, ed. by Einar Anderson. 13 S. New York 1957.

\section{Ubersetzungen b) ins Deutsche}

Warum nennen wir uns Lutheraner? Ubersetzt von Reuter.

Riethmüller, Otto: Übersetzung von „Kirken den er et gammelt Hus“: Ewig steht fest der Kirche Haus. Evangelisches Kirchengesangbuch. Norddeutsche Ausgabe. Vgl. Georg Simon, S. 145f.

Warum ist des Herrn Wort aus dessen Hause verschwunden? Eine ProbePredigt von Nic. Fried. Sev. Grundtvig, Kandidat der Theologie. Nürnberg 1811. 24 S. auch: Hadersleben 1812.

Warum werden wir Lutheraner genannt? Eine Predigt am Tage aller Heiligen von Nic. Fried. Sev. Grundtvig. Nürnberg 1815. 63 S. Rez.: Jung-Stilling in: „Der Graue Mann“, Stück 29.

Eidora Taschenbuch auf das Jahr 1823. Erster Jahrgang. Herausgegeben von H. Gardthausen. Schleswig 1823, 415-429 (Übersetzung von „Napoleon Bonaparte" aus "Danne-Virke“ III).

Protest der christlichen Kirche gegen den Afterprotestantismus des Dr. H.N. Clausen von N. F. S. Grundtvig. Übersetzt von H. Egge. Leipzig 1825. 95 S. S. 71-95 Nachschrift des Übersetzers.

Fr. Schlegel: Ueber nordische Dichtkunst. In: „Deutsches Museum“. Wien 1812 = Sämmtliche Werke X, 1825, 92-95. (Übersetzung eines Abschnittes aus Nordens Mythologie).

N.F.S. Grundtvigs Politische Betrachtungen mit einem Blick auf Dänemark und Holstein. Deutsch hrsg. von C. Harmsen. Kopenhagen 1831. 80 S.

Über die Clausen'sche Injuriensache. Deutsch von C. Siemonsen. Kopenhagen 1832. $16 \mathrm{~S}$.

L. Siemonsen: Geistliche Gesänge und Lieder. Kopenhagen 1832, 200-204, 20918 (Übersetzung von De hellige tre Konger) Der Kämmerer aus dem Mohrenland).

Der Taufbund. Aus dem Dänischen. Hamburg 1836. 32 S. (vgl. Bergedorfer Bote 22/10 1836).

Die Morgendämmerung. 1. Predigt über die Epistel am ersten Sonntage des 
Advents: Röm. 13, 11-14. von N.F.S. Grundtvig. Aus dem Dänischen ins Deutsche übertragen von Dr. A. G. Rudelbach, Superintendeent.

In: Chr. Ph. H. Brandt: Evangelisches Prediger-Magazin. Dritter Band (Zur vergleichenden Homiletik), Sulzbach 1837. Erste Abtheilung. S. 3-15.

Bis hierher hat der Herr sich selbst geholfen. 2. Predigt über das Evangelium am ersten Osterfeiertage: Marc. 16, 1-8. von N. F. S. Grundtvig. Aus dem Deutsche übertragen von Dr. A. G. Rudelbach, Superintendent.

In: Chr. Ph. H. Brandt: Evangelisches Prediger-Magazin. Dritter Band Zur vergleichenden Homiletik), Sulzbach 1837. Erste Abtheilung. S. 14-25.

Grundtvigs Übersicht der Welt-Chronik, vornämlich des lutherischen Zeitraums, nach der Ausgabe von 1817 aus dem Dänischen übertragen von Dr. Volkmann. Durchgesehen und mit einigen Anmerkungen begleitet von Dr. A. G. Rudelbach. Nürnberg 1837. XVI + 464 S.

Nik. Fred. Sev. Grundtvig, Vom wahren Christenthum. Als Gegengift gegen Dr. Karl Gottl. Bretschneiders „religiöse Glaubenslehre nach der Vernunft und der Offenbarung für denkende Leser“. Aus d. Dänischen von Emil Francke. Leipzig 1844. $167 \mathrm{~S}$.

Das Dannewirke-Lied nach Kok und Grundtvig, von A. Gerner. Copenhagen 1863. $4 \mathrm{~S}$.

Album Nordgermanischer Dichtung. Deutsch ... von Edmund Lobedanz. 1. Ausg., Leipzig 1868. S. 82-84 Jaordet) S. 84-86 („Moders navn er en himmelsk Lyd“).

Schumacher, E.: Perlen geistlichen Volksgesangs aus dem Norden Göttingen 1880. $24 \mathrm{~S}$.

Ausgewählte Gedichte von Björnstjerne Björnson ... von E. Lobedanz. Leipzig 1881. S. 194f. (Kirkeklokken).

(Predigten zum 2. Sonntag nach Ostern und zu Trinitatis) In: Aus dem evangelischen Norden. Zeugnisse von Christo in Predigten aus der skandinavischen Kirche unserer Zeit. Úbersetzt und hrg. von O. Gleiss, 1882, S. 201-205 und 240-246.

Grundtvig über seine Volkshochschule. Volksbildungsarchiv VIII, 1922, 241250.

N. F. S. Grundtvig. Schriften zur Volkserziehung und Volkheit. Ausgew., übers. u. eingel. v. Johannes Tiedje. 2 Bde. Jena 1927.

1. Die Volkshochschule (LXXIV, 331 S.).

2. Volkheit (V, $438 \mathrm{~S}$.).

Aaholm, Th. und Westergaard Jacobsen: Deutsch-dänisches Liederbüchlein. Padborg 1960. $16 \mathrm{~S}$. (Ưbersetzungen von Grundtvig-Liedern).

Grundtvigs Volkshochschule. Langensalza 1930. $98 \mathrm{~S}$. = Kleine pädagogische Texte H. 9.

N.F.S. Grundtvigs Volkshochschule (Einf. Erich Weniger). Berlin, Langensalza, Leipzig 1951. $97 \mathrm{~S}$. = Kleine pädagogische Texte 9. 2. Aufl. Weinheim 1962.

Gedichte über Ansgar von N. F. S. Grundtvig. Ubersetzt von Anders Øster. Ansgar und Dänemark. Hrsg. von H. Høirup. Aarhus 1965, 23-56.

Grundtvig auf Deutsch. Eine neue Auswahl von Kirchenliedern Grundtvigs verdeutscht von Werner Görnandt. Helsingör 1967. $15 \mathrm{~S}$. 


\section{Ubersetzungen c) ins Französische}

Simon, Erica: De l'union culturelle de Nord. Texte original ... Traduction et commentaire précédés d'une introduction à la philosophie de la culture de N. F. S. Grundtvig. Kopenhagen 1962. 220 S.

\section{Übersetzungen d) ins Holländische}

Handelingen von den eersten skandinavischen Kerkdag ... Schoonkoven 1858. 246 S. (Bericht mit Reden Grundtvigs).

\section{Monographien über Grundtvig}

Allen, Edgar L.: Bishop Grundtvig: A prophet of the North. London 1949. 94 S. Davies, Noëlle: Education for life. A Danish pioneer. London 1931. 207 S.

Davies, Noëlle: Grundtvig of Denmark. A guide to small nations. Liverpool 1944. $56 \mathrm{~S}$.

Engberg, Poul: N.F.S. Grundtvig. Ein nordischer Volkserzieher. Deutsch von Victor A. Schmidtz. Stuttgart 1950. 99 S. = Erziehungswissenschaftliche Bücher. Reihe 1: Gedanken führender Pädagogen.

Görnandt, Werner: Grundtvig als Kirchenliederdichter in lutherischer und ökumenischer Sicht. Kopenhagen 1963. 58 S. 2. erweiterte Aufl., Helsingør 1969. $80 \mathrm{~S}$.

Hansen, Christian: Wesen und Bedeutung des Grundtvigianismus in der dänischen Kirche. Nach seinem Tode herausgegeben von K. Wieseler. Kiel 1863. VII, $124 \mathrm{~S}$.

Harbsmeier, Götz: Wer ist der Mensch? Grundtvigs Beitrag zur humanen Existenz. Alternativen zu Kierkegaard. Göttingen 1972. $245 \mathrm{~S}$.

Høirup, Henning (Hrsg.): Ansgar und Dänemark. Grundtvigs Beitrag zum Gedächtnis Ansgars. Herausgegeben am 1100jährigen Gedenktag an Ansgars Tod, dem 3. Februar 1965, von Henning Høirup. Aarhus 1965. $56 \mathrm{~S}$.

Holm, Sören: Grundtvig und Kierkegaard. Parallelen und Kontraste. Kopenhagen und Tübingen 1956. $103 \mathrm{~S}$.

Kaftan, Julius: Grundtvig, der Prophet des Nordens. Zwei Vorträge. Basel 1876. $35 \mathrm{~S}$.

Knudsen, Johannes: Danish Rebel. The Life of N. F. S. Grundtvig. Philadelphia 1955. XIII, $242 \mathrm{~S}$.

Koch, Hal: Grundtvig, Leben und Werk. Aus dem Dänischen v. H. Winkler und Victor A. Schmitz. Köln und Berlin 1951. $211 \mathrm{~S}$.

Koch, Hal: Grundtvig. Transl. from the Danish with introduction and notes by Llewellyn Jones. Yellow Springs, Ohio 1952. $231 \mathrm{~S}$.

Koch, Hal: Nicolas-Frédéric Grundtvig, 1783-1872, barde et animateur du peuple danois. Genève 1944.

Koch, Hal: Nikolai Frederik Severin Grundtvig, 1783-1872. Aus dem Dän. ins Dt. übertr.: Grete Schwemm. Bremen 1960. 31 S. = Bremer Beiträge zur freien Volksbildung H. 4.

Lehmann, Edvard: Grundtvig. Aus dem dänischen übersetzt von Anders Oster. Mit einer biographischen Einleitung von Valdemar Ammundsen. 
Tübingen 1932. 72, 282 S. Rez.: Die Christliche Welt XLVIII, 1934, 398406 (R. Buchwald).

Lindhardt, P. G.: Grundtvig. An Introduction. London 1951. 141 S. Rez.: Orbis Litterarum IX, 1954, 247-49 (Helge Toldberg).

Lorentzen, Johs.: Diesseits und jenseits der Grenze. Nicolai Frederic Severin Grundtvig und Claus Harms. Gegenwartsfragen im Lichte der Vergangenheit. Neumünster 1933. $136 \mathrm{~S}$.

Madsen, Georg Rönberg: Bischof N.F.S.Grundtvig und seine Bedeutung als Pädagog (Diss. Jena). Langensalza 1905. $125 \mathrm{~S}$.

$=$ Grundtvig und die dänische Volkshochschule. Pädagogisches Magazin H. 253. Langensalza 1905. $124 \mathrm{~S}$.

Marais, J. I.: Bishop Grundtvig and the people's high school in Denmark. Pretoria 1911. $38 \mathrm{~S}$.

Neuman, St. Elmo H. (Jr.): The Social Philosophy of Søren Kierkegaard and Nikolai Frederik Severin Grundtvig. 224 S. Ph. D. thesis. Boston University Graduate School. 1969.

cf. Dissertation Abstracts XXX A, 2081f.

Nielsen, Ernest D.: N. F. S. Grundtvig: An American study. Rock Island 1955. XII, $173 \mathrm{~S}$.

Schaeffer, Frank L.S.: Untersuchungen über Grundtvigs Entwicklung zum Prediger und seine christliche Auffassung mit besonderer Bezugnahme auf seine letzten Predigten (Diss. Greifswald). Kopenhagen 1944. 196 S.

Simon, Erica: Réveil national et culture populaire en Scandinavie. La genese de la højskole nordique 1844-1878 (Thèse). Paris 1960. $766 \mathrm{~S}$.

Simon, Erica: De l'union culturelle de Nord. Texte original ... Traduction et commentaire précédés d'une introduction à la philosophie de la culture de N. F. S. Grundtvig. Kopenhagen 1962. 220 S.

Simon, Georg: Sucher, Prediger, Dichter.

Nikolai F. Grundtvig. Erneuerer der dänischen Kirche. Berlin 1965. 152 S.

Sontag, Elisabeth: N. F. S. Grundtvig. Erzieher seines Volkes. Bern 1946 (Thèse, Genève). 131 S. Rez.: Pädagogisches Archiv II, 1949, Nr. 1, S. 11-12. (Ernst Jungmann).

Thaning, Kaj: Der Däne N. F. S. Grundtvig. Übers. v. Eberhard Harbsmeier. 184 S. - Auch auf English und Französisch erschienen. - Kopenhagen 1972.

Wartenweiler-Haffter, Fritz: Ein nordischer Volkserzieher. Die Entwicklung N. F. S. Grundtvigs zum Vater der Volkshochschule. Bern 1913. 219 S.

\section{Aufsätze in Zeitschriften und Sammelwerken. Behandlung Grundtvigs in anderen Werken}

Aaberg, J. S.: Danish Hymns and Hymn-Writers. Des Moines: The Committee of Publications of the Danish Evangelical Lutheran Church of America 1945.

Allchin, A. M.: Grundtvig's Translation from the Greek. The Eastern Churches Quarterly XIV, 1961-62, 28-44.

Allchin, A. M.: The Hymns of N.F.S. Grundtvig. The Eastern Churches Quarterly XIII, 1959, 129-43. 
Allen, E. L.: Grundtvig and Kierkegaard. Congregational Quarterly XXIV, London 1946, 205-12.

Andersen: Grundtvig. Vortrag. S. Hol. Kirchen- und Schulbl. 1887, 173-76, 177-79, 181-82, 183-85.

Aronson, Harry: Grundtvigs gedachten over kerk en school. Vox Theologica. Interacademiaal Theologisch Tijdschrift. Assen 1961, 117-124.

Begtrup, H.; Lund, H., Manniche, P.: The Folk High Schools of Denmark and the development of a farming community. 4. ld. London 1948. $163 \mathrm{~S}$.

Billeskov Jansen, F. J.: L'Age d'Or Deux conférences sur la littérature classique du Danemark. Kopenhagen 1953, S. 28-33.

Boje, Andreas, D. J. Borup \& H. Rützenbeck: Education in Denmark. The intellectual basis of a democratic commonwealth. London \& Kopenhagen 1932. $291 \mathrm{~S}$.

d. Boor, H.: Art. Grundtvig in: RGG² II, 1519-1521.

Borelius, Hilmar: (Grundtvig) Die Nordischen Literaturen. Handbuch der Literaturwissenschaft, hrg. von Oskar Walzel. Potsdam 1931, S. 61-63.

Borup, Ernst J.: Grundtvigianismus und Volkshochschule in Dänemark. Ekklesia II. Die Kirche in Dänemark. Leipzig 1937, 142-151.

Bredsdorff, Elias: Danish Literature in English Translation. Orbis Litterarum V, 1947, 187-257 (S. 216f. über Grundtvig).

Bredsdorff, Elias: Danish Literature in English Translation. A bibliography. Kopenhagen 1950. S. 60-61 über Grundtvig.

Bredsdorff, Elias: Grundtvig in Cambridge. The Norseman. March-April 1952. 114-123.

Buchwald, R.: Eine deutsche Grundtvig-Ausgabe. Diederichs Löwe II, 1. H., 1928, 47-50.

Buchwald, Reinhard: Grundtvig und die dänische Volkshochschule. Die Christliche Welt XLVI, 1932, 58-65.

Burger, J.F. (Johannes Fransois): Vergleichende Untersuchungen über die Volkshochschulbewegungen in Dänemark, England und Deutschland. München 1926. $95 \mathrm{~S}$. (Phl. Diss.).

Buss, Hinrich: Das Menschliche und die Existenz. Grundtvig und Kierkegaard. Kontroverse I, 1966, 81-93.

Cambell, Olive Dame: The Danish Folk School. New York 1928. 359 S.

Christmas Møller, J. und Watson, Katherine: Education in democracy. The Folk high schools of Denmark. London 1944. $160 \mathrm{~S}$.

Cogordan, G.: Revue des deux Mondes XIII, 1876, 524-554.

Cooley, E. G.: Bishop Grundtvig and people's high schools. Educational Review, New York, XLVIII, 1915, 452.

Cooley, Franklin: Grundtvig's first translation from Beowulf. Scand. Studies and Notes XVI, 1940, 234-38.

Desmond, Shaw: The Soul of Denmark. London 1918. 277 S.

Dietrich, A.: Grundtvig. Gewissen X, 1928, Nr. 11.

Driffield-Hawkin, T.: When Grundtvig worked at Exeter. „Denmark“, London, Aug. 1948.

Durand, Frédéric: (Grundtvig) Historie de la littérature danoise. KopenhagenParis 1967, S. 155-161. 
Durand, Frédéric: (Grundtvig) Historie génerale des littératures II. Paris 1961, S. 720-722.

Egglishaw, Jan Jepson: „The Land of the Living“. The Norseman, March-April 1952, $124 \mathrm{f}$.

Erb, Jörg: Nikolaus Friedrich Grundtvig. Die Wolke der Zeugen, Kassel 1951, 453-59.

Fauerholdt, J. V. R.: Schulgedanken bei Grundtvig. Neue kirchliche Zeitschrift XL, 1929, 405-14.

Flitner, Wilhelm: Grundtvig, der nordische Gedanke und die deutsche Geistesbewegung. Der Wagen 1938, 90-96.

Flitner, W.: Grundtvig und die dänische Volkshochschulbewegung. Zwischen Elbe und Skagerak, hrg. von R. H. Carsten, Hamburg 1938, 173-178.

Foght, H. W.: The Danish folk high schools. Washington 1914. 93 S.

Friberg, Maikki: Entstehung und Entwicklung der Volkshochschulen in den nordischen Ländern. Bern 1901.

Bern 1897. 166 S. (Ethisch-sozialwissenschaftliche Universalbibliothek Bd. 2).

Goodhope, Nana: Christen Kold. The Little Schoolmaster Who Helped Revive a Nation. Blair, Nebraska, 1956, 120 S.

Gosse, Edm. W.: Four Danish Poets (i. e. Grundtvig, Bødtcher, H. C. Andersen and Fr. Paludan Müller).

In Edm. W. Gosse: Studies in the Literature of Northern Europe. London 1879. Reprinted 1883.

Gulińska, Zofja: Grundtvig wychowawca narodu duńskiego. Referat. Warszawa 1927. $32 \mathrm{~S}$.

Hamdorff, G.: Der Volkserwecker N. S. F. Grundtvig, 1783-1872. Geisteskultur und Volksbildung XXIX, 1920, 3-9.

Hansen, Marie: Grundtvig, l'éducateur du peuple danois. La revue du christianisme social XLIII, 1931, 180-98.

Harbsmeier, Götz: Kierkegaard und Grundtvig. Ev. Theol. XXV, 1965, S. 7282. Wieder abgedruckt in: Kontroverse um Kierkegaard und Grundtvig I, München 1966, 94-108, und in: Sören Kierkegaard, Hrg. Heinz-Horst Schrey. Wege der Forschung Band CLXXIX, Darmstadt 1971, 385-399.

Hart, Joseph K.: Light from the north. The Danish folk high schools. Their meanings for America. New York 1927, $159 \mathrm{~S}$.

Henningsen, A.: Grundtvig. Deutsches Volkstum. Monatsschrift X, 1927, 193199.

The Danish People's High School. Publ. by „The Association of People's High Schools and Agricultural Schools". Transl. by Shaw Desmond. Kopenhagen 1918. $169 \mathrm{~S}$.

Hildebrandt, Else: Die bleibenden Werte der Bildungsideale N. F. Grundtvigs. Monatshefte der Comenius-Gesellschaft für Kultur und Geistesleben, N. F. Bd. 7, Jena 1915, 163-168.

Hirsch, Emanuel: (Grundtvig) Geschichte der neuern evangelischen Theologie, Band V. Gütersloh 1954, S. 221-231.

Høirup, Henning: Grundtvigs Gedanken über Christentum und Volk. Grundtvig Studier 1952, 72-81.

Høirup, Henning: Grundtvig and Kierkegaard, their views on the church. 
Theology today XII, 1955, 328-342. Wieder abgedruckt in: Grundtvig Studier 56, 205-12.

Høirup, Henning: Grundtvigs Beitrag zum Gedächtnis Ansgars. Ansgar und Dänemark, 7-19.

Holdt, Jens: Grundtvigs nordisches Christentum. Die Christliche Welt LIII, 1939, 93-100.

Hollmann, A.H.: Die dänische Volkshochschule und ihre Bedeutung für die Entwicklung einer völkischen Kultur in Dänemark. Berlin 1909. 2. Aufl. 1919 unter dem Titel: Die Volkshochschule und die 'geistigen Grundlagen der Demokratie. $143 \mathrm{~S}$.

Holm, Sören: Grundtvig und Kierkegaard, Parallelen und Kontraste. Zeitschrift für systematische Theologie XXIII, 1954, 158-176.

Holm, Sören: Mythus und Kult in Grundtvigs Kirchenliederdichtung. Zeitschrift für systematische Theologie XXIV, 1955, 17-37.

Holm, Sören: Holberg, Grundtvig, Kierkegaard, drei dänische Denker. Neue Zeitschrift für systematische Theologie VI, 1965, 49-61.

Hunkel, E.: Grundtvig, Volkserzieher des Nordens, und seine Beziehung zum deutschen Geistesleben. Jahrbuch der deutschen Volkshochschulbewegung I, Hellerau 1919, 9-15.

Ihlen, Chr.: "Das goldene Zeitalter" Dänemarks in Norwegen. Zeitschrift für systematische Theologie XIII, 1936, 525-41.

Janson, Kristoffer: Grundtvig and the Peasant High School. Scandinavia. Chicago 1884.

Jessen, E. A. F.: Die Hauptströmungen des religiösen Lebens der Jetztzeit in Dänemark. Gütersloh 1885. $176 \mathrm{~S}$.

Jørgensen, Johannes: Geschichte der dänischen Literatur. Kempten und München 1908. 157 S. (Sammlung Kösel 19) S. 89-94.

Jones, Llewellyn: Kierkegaard or Grundtvig? Christ. Century LXIX, 1952, n. 20, 588-89. cf. ibid. n. 23, 674-75.

Kautz, H.: Nicolai Frederic Grundtvig und die deutsche Pädagogik. Pharus. Katholische Monatsschrift XXIII, 1932, 456-459.

Søren Kierkegaard: Unwissenschaftliche Nachschrift. Erster Teil § 2: Von der Kirche. Hegner-Ausgabe S. 162-176.

Klein, E. F.: Zwei Dänen, Kierkegaard und Grundtvig. Zeitbilder aus der Kirchengeschichte für die christliche Gemeinde, Bd. II, Berlin 1927, S. 81ff.

Knudsen, Johannes: Grundtvig Research. The Lutheran Quarterly V, 1953, 167-174.

Knudsen, Johannes: Grundtvig and Theology. The Lutheran Quarterly VI, 1954, 299-309.

Knudsen, Johannes: Grundtvig und die heutige amerikanische Theologie. Lutherische Rundschau IV, 1954/5, 306-318.

Knudsen, Johannes: Grundtvig and American Theology Today. Lutheran World I, 1954/5, 277-287.

Knudsen, Johannes: Notes about Grundtvig. Summer Issue of The Chicago Lutheran Theological Seminary Record (Juli 1955).

Knudsen, Johannes: Revelation and man According to N. F. S. Grundtvig. Lutheran Quarterly X, 1958, 217-225. 
Koch, Hal: Art. Grundtvig, Grundtvigianismus. In: RGG³ II, Sp. $1894 \mathrm{f}$.

Kosmehl, K.: Grundtvig, sein Leben und seine Bedeutung. Die völkische Schule, Breslau, I, 1933, 308-315.

Lecarpentier, G.: Les hautes écoles du peuple danois. Annales des Sciences Politiques. Revue bimestrielle, 21. Jahrgang, 15. November 1906.

Lehmann, Edvard: Grundtvig. Zum 50-jährigen Gedenktag seines Todes. Die christliche Welt XXXVI, 1922, 845-48.

Lehmann, Ed.: Deux réformateurs du protestantisme danois: Sören Kierkegaard et Grundtvig. Revue d'historie et de philosophie religieuses 1931, S. 499505.

Lembke, Fr.: Die Dänische Volkshochschule nebst Plan einer Deutschen ländlichen Volkshochschule. Kiel und Leipzig 1904 (Sonderheft 1 der Zeitschrift für das gesamte Fortbildungsschulwesen in Preussen) $55 \mathrm{~S}$.

Lindhardt, P. G.: Grundtvig and England. Journal of ecclesiastical history I, 1950, 207-224.

Løgstrup, Knud E. und Götz Harbsmeier (Hrg.): Kontroverse um Kierkegaard und Grundtvig I: Das Menschliche und das Christliche. München 1966. $140 \mathrm{~S}$.

Lüttke, Moritz: Kirchliche Zustände in den skandinavischen Ländern, Dänemark, Norwegen, Schweden. Elberfeld 1864. 144 S.

Lund, Ragnar (ed.): Scandinavian Adult Education. Editor in chief: Ragnar Lund. First Edition 1949, second Edition 1952. 297 S.

Mac Kaye, David L.: Grundtvig and Kold. The American-Scandinavian Review III, 1942, S. 229-239.

Magelund, Johannes: Grundtvig und die christlichen Volkshochschulen in Skandinavien. Kirche im Dorf IX, 1958, 296-299.

Malone, Kemp: Grundtvig's philosophy of history. Journal of the hist. of ideas I, 1940, 281-298.

Malone, Kemp: Grundtvig on Paradise Lost. Renaissance Studies in Honor of Hardin Craig, 1941, 320-323.

Malone, Kemp: Grundtvig as Beowulf Critic. The Review of English Studies XVII, 1941, 129-138.

Manniche, Peter: Living Democracy in Denmark. Rev ed. of „Denmark, a social laboratory". Kopenhagen 1952. $240 \mathrm{~S}$.

Melching, L.: Grundtvigs Schriften zur Volkserneuerung. Volk und Heimat, hrg. von Verband für nationale Volkserziehung München, IX, 1933 Nr. 22, S. 3.

Mentzel, H. G.: Glaube und Volkstum. Zum Gedenken an Nikolaus Frederik Grundtvig, 1783-1872. Stettiner Nachrichten, Göttingen, XIII, 1962, Nr. 9, S. 10.

Mesnard, Pierre: Philosophie de l'histoire et pédagogie chez Nicolas-Frédéric Grundtvig (1783-1872). Etudes Germaniques XVIII, 1963, 328-43.

Meyersahm, H.: Grundtvig und Claus Harms. Zwei streitbare Kirchenmänner. Eiserne Blätter XVI, 1934, 383-387.

Michelsen: Art, Grundtvig in: $\mathrm{RE}^{2} \mathrm{~V}, \mathbf{4 3 9 - 4 5 0 .}$

Moe, Olaf: 10jähriges Gedächtnis des Dänen N. F. S. Grundtvig. Allgem. evang.luth. Kirchenzeitung 1925, 975, 1000.

Monrad, O. P.: Der Grundtvigianismus. Die Christliche Welt 1901, Nr. 23, 24. 
Müller, Carl: Grundtvig und die Heimvolkshochschule in Dänemark. Germanica 24.9. 1927.

Nägele, Horst: Von einem „Echten Deutsch“ und von der „Deutschen Sprache des Herzens". Feststellungen des frühen N. F.S. Grundtvig zur Überbaufunktion eines sogenannten Hochdeutsch als übergreifende Institution. Grundtvig Studier 1971, 74-82.

Naumann, P.: Grundtvig, ein nordischer Volkserzieher. Allgemeine deutsche Lehrerzeitung LXII, 1933, 644.

Nielsen, Ernest D.: N. F.S. Grundtvig on Luther. Interpretations of Luther. Essays in Honor of Wilhelm Pauck. Ed by Jaroslav Pelican, Philadelphia 1968, S. 159-186.

Nielsen, Ernest D.: „Thomas Chalmers“. A study of selected writing of Chalmers to determine whether Grundtvig was influenced by Thomas Chalmers. Lutheran Tidings, VIII, 5. Dez. 1930 u. 20. Dez. 1930.

Ostergaard Nielsen, Harald: Das Erbe Kierkegaards und Grundtvigs im Widerstreit. Kontroverse I, 1966, 13-49.

Paul-Dubois, L.: Grundtvig et le relevement du Danemark. Revue des Deux Mondes LII, 1909.

Pentzlin, J.: N. F. S. Grundtvig. Der alte Glaube IV, 1902/3, Nr. 27-29.

Perger, A.: N. F. S. Grundtvigs religiöse Anschauungen und Wandlungen. Der katholische Seelsorger 1899, 413-18, 463-67.

Petersen: (Besprechung einiger Schriften Grundtvigs). Schleswig-Holstein- Lauenburgische Provinzialberichte 1818, 763-65.

Prenter, Regin: N.F.S. Grundtvig's sogenannte kirchliche Anschauung als Frage an die evangelische Theologie von heute. Evangelische Theologie I, 1934, 278-288.

Prenter, Regin: Die Frage nach einer theologischen Grundtviginterpretation. Theologische Aufsätze. Karl Barth zum 50. Geburtstag, München 1936, 505-513.

Prenter, Regin: Grundtvigs Ansicht vom Menschen. Evangelische Theologie IX, 1949/50, 395-405.

Prenter, Regin: Schöpfung und Erlösung. Dogmatik. Bd. I - II, Göttingen 1958-60. (Siehe Register).

Puder, H.: Die nordischen Volkshochschulen. Zeitschrift für Philosophie und Pädagogik X, 1903, 22-42.

Rabeler, Werner: Grundtvig zwischen England und Deutschland. Niederdeutsche Welt XV, 1940, 2-4.

Rauch, Chr.: Dänische Dichtung im Kampfe gegen Deutschland. „Im neuen Reich“, 1872, S. 841-855.

Reilingh, H. D. de Vries: Nikolai Frederik Grundtvig en onze tijd. Het Gemeenbest. Haarlem, Amsterdam, IV, 1941/2, 267-74.

Rein, Wilhelm: Von den dänischen Volkshochschulen. Die Gegenwart. Wochenschrift für Literatur. Kunst und öffentliches Leben XLVII, 1895.

Rieken, W.: N. F. S. Grundtvig, der Vater der Volkshochschulidee. Pädagogische Warte 1910, 1167, 1221-29.

Rørdam, Thomas: The Danish Folk High Schools. Det Danske Selskab 1965. $199 \mathrm{~S}$. 
Rosbach, P.: Grundtvig. Pädagogisches Zentralblatt, Langensalza, XIII, 1933, 342-64.

Rosenberg, C.: Almanach populaire illustré de la Suisse Romande pour 1871, S. $61 \mathrm{ff}$.

Rosenberg, P. A.: Grundtvig. American-Scandinavian Review XXI, 1933, 482-91.

Rudelbach, A. G.: (Grundtvig) in: Erschs und Grubers Enzyclopädie 1. Section XXIX, S. 96 u. 98 (1837) XCV (1875), S. 225-26.

Rudelbach, Andreas Gottlob: Das Christenthum und die Rationalisten in Dänemark. Evangelische Kirchenzeitung 1827, Nr. 51-52; 1828, Nr. 55-57, 62, $63 ; 1830$, Nr. $5-8 ; 1831$, Nr. 69, 70, 73-75; 1832, Nr. 49-51; 1835 , Nr. 26-33.

Rudelbach, Andreas Gottlob: Beiträge zu einer Charakteristik der letzten religiösen Erweckung in der evang. luth. Kirche Dänemarks mit Bezugnahme auf die kirchlichen Zustände dort seit 1836. Zeitschrift für die gesamte Lutherische Theologie und Kirche II, 1841, 65-115, 83-130.

Savage, David J.: Grundtvig: A stimulus to old english scholarschip. Philologica: The Malone Anniversary Studies. Ed. by Thomas A. Kirby and Henry Bosley Woolf. Baltimore 1949, 275-280.

Scharling, C. I.: Grundtvigs Bedeutung für das dänische Gemeindeleben. Monatsschrift für Pastoraltheologie XXX, 1934, 129-156.

Scheel, Otto: Stromkenterung in den deutsch-dänischen Beziehungen. Jomsburg $\mathrm{V}, 1941,151-163$.

Schmitz, Victor A.: Der Gedanke vom lebendigen Wort bei N. F. S. Grundtvig. Zeitschrift für systematische Theologie XI, 1934, 75-107.

Schröder, L.: Art. Grundtvig. In: RE ${ }^{3}$ VII, 206-217.

Simon, Erica: L'universalité de Grundtvig. Études Germaniques XXII, 1967, 445-48.

Simon, Erica: Grundtvig et la France. Grundtvig Studier 1969, 7-22.

Skrubbeltrang, Fr.: The Danish folk high schools (Danish information Handbooks). 2. ed. Revised by Roar Skovmand, 1952. $88 \mathrm{~S}$.

Skarsten, Trygve R.: Rise and fall of Grundtvigianism in Norway. Lutheran Quarterly XVII, 1965, 122-42.

Steffen, G.: Art. Grundtvig. In EKL I, Sp. 1729-1731.

Steffen, Gregor: Aus Grundtvigs Leben und Werk. Ev.-luth. Kirchenzeitung III, 1949, 136-137.

Steffensen, Steffen: Grundtvig und Kierkegaard. Ausblick, Lübeck 1950, 49-50.

Steffensen, Steffen: Grundtvig und die deutsche Romantik. Beiträge zur deutschen und nordischen Literatur. Festgabe für Leopold Magon zum 70. Geburstag. Berlin 1958, 282-290.

Strodtmann, Adolf: Das geistige Leben in Dänemark. Streifzüge auf den Gebieten der Kunst, Literatur, Politik und Journalistik des skandinavischen Nordens. Berlin 1873. 339 S.

Svendsen, Hanne Marie und Werner: (Grundtvig) Geschichte der dänischen Literatur. Neumünster und Kopenhagen 1964, S. 191-195.

Thaning, Kaj: Die dänische Volkshochschule. Junge Kirche XXI, 1961, 65-75.

Thaning, Kaj: Die dänische Volkskirche. Junge Kirche XXI, 1961, 137-147.

Thaning, Kaj: „Man first". Danish Foreign Office Journal, nr. 48, 1964, 29-33. 
Thaning, Kaj: Das Menschliche und das Christliche bei N.F.S. Grundtvig. Kontroverse I, 1966, 50-80.

Toldberg, Helge: Zur Holsteinischen Reimchronik. Philologica: The Malone Anniversary Studies. Ed. by Thomas A. Kirby and Henry Bosley Woolf. Baltimore 1949, 275-280.

Totomianz, V.: Nikolai Frederik Grundtvig. Raiffeisen-Rundschau V, 1953, Nr. 4, 85-86.

Trummler, E.: Grundtvigs Hochschulidee. Ist sie verwirklicht worden? Goetheanum. Antroposophie XIV, 1935, 195-197.

Vaaben, E.: N. F.S. Grundtvig. Rasse. Monatsschrift der nordischen Bewegung, Leipzig, VI, 1939, 89-100.

Wartenweiler-Haffter, Fritz: Aus der Werdezeit der dänischen Volkshochschule. Das Lebensbild ihres Begründers Christen Mikkelsen Kold. ErlenbachZürich 1921. 93 S. 2. Aufl.: Ein Sokrates in dänischen Kleidern. Christen Kold und die erste Volkshochschule. Zürich 1929. $101 \mathrm{~S}$.

Wartenweiler-Haffter, Fritz: Von der dänischen Volkshochschule. Ein Erlebnis. Zürich 1921. $77 \mathrm{~S}$.

Wartenweiler-Haffter, Fritz: Artikel: Grundtvig in: Lexikon der Pädagogik III, Bern 1952, S. 185-186.

Waschnitius, V.: N.F.S. Grundtvigs Bedeutung für die Bildung des dänischen Geistes. Deutsche nordische Zeitschrift I, 1928, 3-18.

Weniger, E.: Grundtvig. Die Erziehung V, 1930, 193-218, 280-294. = Weniger, E.: Grundtvig und der Begriff der historischen Aufklärung.

In: Weniger, E.: Die Eigenständigkeit der Erziehung in Theorie und Praxis. Probleme der akademischen Lehrerbildung. Weinheim 1952, S. 172-215.

Werner, Joh.: Art. Grundtvig, in: RGG $\mathbf{1}$ II, 1720-23.

Winkel-Horn, Frederik: Geschichte der Literatur des Skandinavischen Nordens von den ältesten Zeiten bis auf die Gegenwart. Leipzig 1880. IX, 404 S.

Winkel-Horn, Frederik: History of the Literature of the Scandinavian North from the Most Ancient Times to the Present. Translated by Rasmus B. Anderson. Chicago 1884. IX 3507 S. (S. 242-248 über Grundtvig).

Winther, Chr.: Grundtvig und seine Bedeutung für die Gemeinde in Dänemark. Allgemeine ev.-luth. Kirchenzeitung 1929, 496.

(Anon.): Nikolai Frederik Severin Grundtvig. Neue evangelische Kirchenzeitung, 1872, 753-56.

(Anon.): Esaias Tegnér und Nic. Fred. Sev. Grundtvig. Evangelische Kirchenzeitung, 1873, 1129-42.

(Anon.): Grundtvig und die dänische Volkshochschule. Schweizerische Lehrerzeitung, 1919, S. 85, 95.

(Anon.): Grundtvig, der Prophet des Nordens. Deutsche Bauernhochschule, Hellerau 1921 (Hakenkreuzverlag), 1. H., S. 32.

(Anon.): Grundtvig und die Gründung der dänischen Volkshochschule. Schweizer Volkshochschule III, 1924, 98-103.

(Anon.): Zur Charakteristik des Grundtvigianismus. Allgem. evang.-luth. Kirchenzeitung XX, 130.

(Anon.): Grundtvig. Zu seinem 150. Geburtstag. Die Hilfe XXXIX, 1933, $450-54$. 


\section{Fremdsprachige Zusammenfassungen in dänischen Werken}

Grundtvig Studier. Kopenhagen 1948 ff. Englische Zusammenfassungen in jedem Band.

Aronson, Harry: Mänskligt och Kristet. Stockholm 1960. Summary in English: S. 294-301.

Bugge, Knud Eyvin: Skolen for livet. Kopenhagen 1965. Summary by Nöelle Davies: S. 361-369.

William Michelsen: Den sælsomme forvandling i N.F.S. Grundtvigs Liv. Kopenhagen 1956. Summary: The strange Metamorphosis in N. F. S. Grundtvig's Life: S. 278-283.

William Michelsen: Tilblivelsen af Grundtvigs Historiesyn. Kopenhagen 1954. Summary: The Genesis of Grundtvig's View of History: S. 349-362.

Kaj Thaning: Menneske først. Kopenhagen 1963. Deutsche Zusammenfassung: S. 777-784.

Die Bibliographie ist 1970 abgeschlossen; einige Titel sind noch später hinzugefügt worden. 